\title{
Antibiotikum hilft selten bei refraktärem Husten
}

\author{
Eine antibiotische Therapie mit Azithromycin hilft in der Regel nicht gegen Husten unklarer \\ Genese. Nur Patienten, die zusätzlich ein Asthma bronchiale haben, scheinen zu profitieren.
}

— Bei hartnäckig therapierefraktärem Husten wird auch ohne Infektnachweis meist irgendwann ein Versuch mit einem Antibiotikum unternommen. 44 solcher Patienten wurden nun in eine englische Studie eingeschlossen. Sie waren Nichtraucher und im Schnitt 59 Jahre alt. Darunter waren auch vortherapierte Patienten mit Zeichen einer bronchialen Hyperreagibilität, mit Reflux oder Rhinitis. Primäres Studienziel war eine Score-Veränderung im Leicester Cough Questionnaire (LCQ), als sekundäre Ziele wurden der Schweregrad des Hustens und die NO-Konzentration in der Ausatemluft untersucht.

Die Patienten erhielten randomisiert entweder $3 \times 250$ Azithromycin oder
Placebo. In der Verumgruppe kam es initial zu einer klinisch bedeutsamen und statistisch signifikanten Verbesserung des LCQ-Scores und der Schwere des Hustens. Die Vorteile ließen sich bei Studienende nach acht Wochen allerdings nicht mehr nachweisen.

In einer Post-hoc-Analyse der klinischen Responder - 11 der 21 Personen aus der Azithromycin-Gruppe - zeigte sich jedoch eine signifikante Assoziation zwischen der Diagnose eines gegenwärtig vorhandenen oder früher diagnostizierten Asthma bronchiale und dem Ansprechen auf das Antibiotikum.

- Hodgson D et al. The effects of azithromycin in treatmentresistant cough: a randomized, double-blind, placebocontrolled trial. Chest. 2016;149:1052-60

\section{KOMMENTAR}

Das Ergebnis, dass bei Patienten mit behandeltem Asthma der Husten mit Azithromycin gut zu beeinflussen ist, kam eher zufällig zustande. Möglicherweise liegt die Erklärung darin, dass die Lungen von Patienten, die inhalative Steroide anwenden, vermehrt mit Streptococcus pneumoniae, Hämophilus influenzae und nicht-tuberkulösen Mykobakterien kolonisiert sind. Diese wiederum könnten für den Husten verantwortlich sein.

Dass die Autoren überhaupt Patienten mit Komorbiditäten eingeschlossen haben, erklären sie mit dem Willen, das tatsächlich in der Praxis auftauchende Patientenkollektiv realistisch abbilden zu wollen.

Prof. Dr. med. H. S. FüeßI

\section{Hier steht eine Anzeige.}

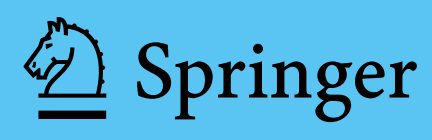

